QVIPUKAMAYOC Revista de la Facultad de Ciencias Contables

Vol. 20 N. 38 pp. 75-86 (2012) UNMSM, Lima, Perú

ISSN: 1560-9103 (versión impresa) / ISSN: 1609-8196 (versión electrónica)

\title{
MIRADA ONTOLÓGICA A LA GESTIÓN DEL CONOCIMIENTO Y SU IMPLICANCIA EN LA EDUCACIÓN
}

\author{
ONTOLOGICAL OVERVIEW TO KNOWLEDGE MANAGEMENT \\ AND ITS IMPLICATIONS IN EDUCATION
}

\author{
Ruth M. Romero Huamani* \\ Docente Contratada de la Facultad de Ciencias Contables \\ Universidad Nacional Mayor de San Marcos-UNMSM \\ Lima-Perú
}

[Recepción: Setiembre de 2012/ Conformidad: Octubre 2012]

\section{RESUMEN}

El artículo trata de construir e implementar un pensamiento crítico sobre la teoría de Gestión del conocimiento como parte de la filosofía, aplicada a la realidad educativa, es decir la forma cómo se percibe desde la mirada ontológica la situación del conocimiento en las organizaciones institucionales. La teoría de gestión es un modelo especulativo que manifiesta una exègesis de las tendencias de mundialización, planetariación y globali-zación, que desde un sentido gnoseológico aborda los problemas curriculares, culturales y políticos en las instituciones educativas. Las culturas productores del conocimiento e información poseen una geografía diseñada para el contexto de la diversidad de saberes, que muchas veces aún están sin explorar. Sin embargo, la mala gestión ha llevado consigo a la desarticulación de la educación, creando una incertidumbre que siguen perennes en el desa-rrollo de la formación de las generaciones. En tanto, la buena gestión es pensar en lo conocido para descubrir lo desconocido, considerando el capital intelectual y organizacional como paradigma de la buena educación y el buabn trato de persona a personas en los buen trato de persona a personas en
política curricular, edyycacion
espacios educativos y lugares públicos.

\begin{abstract}
This article attempts to build and implement a critical thinking about the theory of knowledge management as a part of philosophy, applied to the educational reality, that is the way how you perceive the situation of knowledge, from the ontological point of view, in institutional organizations. The management theory is a speculative model that expresses an exegesis of globalization and planetarization trends, which, from a gnoseological sense, deals with about the curriculum, political and cultural matters in the educational institutions.

Producing cultures of knowledge and information have a geography designed for the context of the diversity of knowledge that often are still unexplored. However, poor management of knowledge has involved the disarticulation of education, creating an uncertainty that remains in developing the forming of generations. Meanwhile, the good management is thinking in the known to discover the unknown, considering the intellectual and organizational capital as a paradigm of good education and good treatment from person to person in the educational and public places.
\end{abstract}

Keywords: Knowledge management, Curricular policy, Education.

* Doctoranda en la Pontificia Universidad Católica del Perú. Magíster en Filosofía por la Universidad Iberoamericana de México, D. F.. E-mail: romeroh24@yahoo.es 


\section{INTRODUCCIÓN}

La globalización es el símbolo de nuestra era como civilización, nos encontramos en un vertiginoso cambio y un progreso invertido de optimismo y pesimismo en las acciones comúnmente vistas en nuestras miradas cotidianas. Tanto como educadores como ciudadanos del mundo. La mundialización para muchos es el darse cuenta de que los seres humanos somos mundanos que tiene historia y conciencia de ella, es decir existentes esencialmente productores de conocimientos e información y aplicación de ella de manera articulada. La planetarización es generalmente geográfica y geopolíticamente tener conciencia del globo terráqueo como parte de la naturaleza humana; aunque aparezcan vertientes y perspectivas que nos lleven a desacuerdos, señalamos lo que comprendemos en la certeza de nuestra racionalidad y entendimiento.

Las tesis más importntes de Castells, Morín, aportes de Elena Cano, CADE y otros, como herramientas de comprensión, nos permiten aproximar y plantear problemas sobre el proyecto de la educación para el Futuro y su búsqueda de horizonte de sentido en la mejora continua de calidad y competencia en la cultura educativa. Para ello, es indispensable responder las siguientes cuestiones: ¿Cuál es o ha sido el impacto de la globalización en el desarrollo de la educación actual, partiendo desde las primeras etapas de la revolución industrial en la modernidad? ¿En qué sociedad vivimos cuando hablamos de la educación en la era planetaria? ¿Por qué los conceptos de globalización, mundialización y planetarización en la Sociedad del conocimiento, que obedecen a la información articulada, ayudan a replantear enfoques medulares basados en los valores y principios en el contexto de la ciudadanía? Y ¿Por qué se habla del capital humano y capital estructural considerando el modus operandi de la lógica del capital intelectual? ¿Cómo detectar los problemas dentro del marco de los capitales en las instituciones, para plantear planes de mejora para una buena educación? Problemas que permiten reflexionar de manera crítica en el marco de los saberes y la naturaleza de la misma en el siglo actual, y nos invita a buscar cambios para una mejor administración, organización y gestión del conocimiento.

\section{DESAFIOS DE LA SOCIEDAD ACTUAL}

Los desafíos son vastas debido a un enorme cambio, transformación y movimiento que presenta la realidad de la sociedad actual, por ello requerimos, en primer lugar un acercamiento conceptual a la globalización. Por un lado, encontramos que la palabra, adaptada a nuestra lengua, tiene un origen en el adjetivo o inglés global que el Compact Oxford English Dictionary lo define de este modo: "global - adjective 1 relating to the whole world; worldwide. 2 relating to or embracing the whole of something, or of a group of things. 3 Computing operating or applying through the whole of a file or program. - DERIVATIVES globalist noun globalize (also globalise) verb globally adverb" (Compact Oxford English Dictionary).

A pesar de que en nuestra lengua global no equivale a mundial como en inglés, el Diccionario de la Real Academia Española registra la entrada globalización, como la "tendencia de los mercados y de las empresas a extenderse, alcanzando una dimensión mundial que sobrepasa las fronteras nacionales" (DRAE 2006); sin embargo, el Diccionario no registra la entrada mundialización. El resaltado es nuestro.

La lectura de esta definición nos muestra la marcada tendencia economicista del 
concepto, así como el hecho tecnológico de las comunicaciones que permitieron el desarrollo de este proceso. Efectivamente, la globalización es un proceso económico, tecnológico, social y cultural que alcanza una dimensión mundial que traspasa las fronteras nacionales. Se caracteriza por la velocidad creciente en la comunicación e interdependencia entre los distintos países que van unificando mercados, ideología, política, tecnología, cultura, etc. La globalización se identifica políticamente con el capitalismo democrático o la democracia liberal y, tecnológicamente, a la revolución informática.

Como lo plantea Alvin Toffler (1980) las ideas globalizadoras y neoliberales nos están trasladando a una tercera ola de civilización en la que la actividad fundamental es el conocimiento y su símbolo es el computador y la Internet.

Ante esta situación, en el crisol de las apariencias abunda en este sentido el concepto de la globalización como un discurso que genera muchas veces una nostalgia interminable en la sociedad. Donde la competencia y calidad ha sido y sigue siendo un paradigma de las comunidades empresariales. Tendencia económica. Ésta tendencia actualmente está siendo tomada como herramienta para repensar y llevar como modelo al sector educativo a nivel mundial, que en los grandes países ya está puesta en funcionamiento y en América Latina aun en proceso, específicamente en el Perú.

Como se constata en los discursos de Castells, Morín, Elena Cano, y aportes de $\mathrm{CADE}$, afirman que el concepto de la globalización hasta hace unos años atrás solamente estaba en la dicotomía de las esferas privadas y públicas de la economía global-empresarial, que de facto por un lado pragmático puesta al servicio de negocios exagerados como: costo y beneficio individual, y por otro en este contexto mismo la inteligencia y la racionalidad humana como mecánico y objeto material, llevada o puesta al servicio del capital como: oferta, demanda y ganancia.

Esto ha permitido el abandono y menosprecio de la capacidad de la inteligencia racional critica y la inteligencia emocional del hombre en el cosmos que alguna vez ha sido demandado ya por el filósofo griego Aristóteles.

Las situaciones mecanicistas, pragmáticas, utilitaristas y economicistas, quizá extremadamente exagerado de parte de un grupo minoritario de la personas abrazadas por la ideología democrática, han dejado la huella encandilado en la conciencia de la humanidad, específicamente en la juventud de hoy, donde prácticamente no se ve la reacción crítica, ni sensibilidad humana con la naturaleza que nos rodea: resultado, la fatal depredación y contaminación al medio ambiente. Crisis de aplicación de criterios de valores morales y otros.

Ante estas realidades y otras, la decadencia de la educación y sus desafíos llaman a gritos en silencio, a que nos ocupemos como ciudadanos del mundo para volver a hacer lo que se ha dejado hacer.

Cuando se habla de las implicancias de la globalización en la educación, según Castells, podemos ver los horizontes de las revoluciones industriales que no sólo ha traído consigo la deshumanización de la propia humanidad, sino también el desarrollo tecnológico como medio para cambiar las formas de vivir la vida. Esto justifica que la tecnología es un medio que depende mucho de sus usos y aplicaciones. Es decir, la innovación tecnológica es neutral. Pero, no cabe duda de que el uso negativo y exagerado de las herramientas tecnológicas haya conducido a la sociedad hacia una barbarie de deslegitimación del sentido humano, como las guerras constantes en el siglo XX. Resultado de estas acciones muestra la ignorancia del hombre 
en el cuidado de la humanidad y su yo, que ya el filósofo Sócrates (V. a C.) en su tesis "conócete a ti mismo", teoría de la verdad y la justicia incorruptible, había denunciado.

La modernidad y sus desafíos consecuentes en la actualidad ha sido una herramienta de la globalización donde las grandes teorías de la ciencia moderna han conducido y colaborado en el holocausto de la rebeldía humana, abandonando la educación, aún sabiendas que ésta es la base fundamental del cuidado entre seres humanos. Educación no es lo mismo que pedagogía como se ha entendido desde los márgenes del cientificismo teórico y metódico, sino educar significa educar al hombre por el hombre para la sociedad desde los márgenes del humanismo articulador.

El siglo XX modelado por la teoría de la relatividad también ha conducido a relativizar las formas de ver la realidad de la educación, por ello el descuido a los infantes (alimentación, educación, salud, y otros) y menosprecio a los recursos naturales (materias primas) de los países subdesarrollados por grandes capitales económicos, que en la actualidad resulta difícil revertir y diseñar propuestas inmediatas para solucionar los grandes desafíos del cambio en el mundo: tanto eventos naturales como artifíciales, es decir la obra del hombre.

No obstante, el surgimiento de las importantes tendencias desafiantes para el cambio en la sociedad contemporánea continúan siendo avalados por los avances científicos y los nuevos desarrollos científico-tecnológicos, nuevas tecnologías para la información y la comunicación (telefonía, informática, etc.), ingeniería genética, nanotecnología y nuevos materiales, tales como:

- Redes de distribución de información de ámbito mundial: Las redes de distribución de información permiten ofrecer en cualquier lugar en el que haya un termi- nal (ordenador, teléfono móvil, televisor) múltiples servicios relacionados con la información. No obstante, lo que para algunos países son grandes y veloces "autopistas de la información", para otros apenas son simples caminos de tierra y barro. Esto significa que la sociedad se basa en amplias redes de comunicación y en la capacidad de los individuos para actualizar su conocimiento en un mundo que cambia vertiginosamente.

- Omnipresencia de los medios de comunicación de masas e Internet: Con los "mass media” (prensa, radio, televisión) e Internet, las noticias de, información, formación y ocio llegan cada vez a más personas. La información se mueve casi con absoluta libertad por todas partes; lo que ocurre en un punto del planeta puede verse inmediatamente en todos los televisores del mundo (hay canales de TV - como la poderosa cadena americana $\mathrm{CNN}$, que emiten noticias, durante todo el día).

Esto supone una verdadera explosión cultural, que hace más asequible el conocimiento a los ciudadanos; pero, a la vez, crece el agobio por el exceso de información y la sensación de manipulación ideológica por los grupos de poder que a través de los "mass media" configuran la opinión pública y afianzan determinados valores.

- Integración cultural: esta es la tendencia que va hacia un "pensamiento único" (sobre todo en temas científicos y económicos), debida, en gran parte, a la labor informativa de los medios de comunicación social (especialmente la televisión), la movilidad de las personas por todos los países del mundo y la unificación de las pautas de actuación que exige la globalización económica.

El sentido de ésta se refuerza en la sensación de pertenecer a una comunidad mundial, aunque los países más poderosos van imponiendo su cultura (idioma, instru- 
mentos y procesos tecnológicos) amenazando la identidad cultural de muchos pueblos.

- La Aceptación del "imperativo tecnológico”: Según este imperativo la fabricación y utilización de herramientas es el factor determinante del progreso de la humanidad. Por ello se aceptan los nuevos instrumentos como modernos e inevitables, renunciando muchas veces a conducir el sentido de los cambios y del progreso. Como afirma Joana Sancho: "las tecnologías artefactuales, simbólicas y organizativas transforman de manera insospechada, no sólo el mundo que nos rodea, sino nuestra propia percepción del mismo y nuestra capacidad para controlarlo"1.

Estas tendencias nos conducen a tomar en cuenta esta metáfora "Hay que tener ordenador y saber inglés".

- Formación de megaciudades: La población se agrupa en grandes aglomeraciones urbanas (megaciudades), donde muchas veces son necesarios desplazamientos importantes para ir a los lugares de trabajo y de ocio como señala el crítico intelectual canadien$\mathrm{se}^{2}$.

- Baja natalidad (en los países desarrollados): En los países desarrollados hay una notable baja de natalidad (Unión Europea), en tanto que en algunos países en desarrollo (China) se toman medidas para contener una tasa de crecimiento excesivo. Mientras otros países siguen sin tomar ninguna medida y con unas tasas de natalidad que desbordan sus posibilidades (México, norte de África).

- Crecimiento del sector servicios en la economía: En un próximo futuro, la gran mayoría de la población activa de la sociedad como tendencia parece que ya no va a estar concentrada en producir alimentos ni en fabricar objetos, sino en ofrecer servicios: procesar información o atender a las personas. Estamos pasando de un consumo fundamentado en los productos a un consumo basado en los servicios, especialmente, en los servicios relacionados con la creación, proceso y difusión de la información.

- Consolidación del neoliberalismo económico: El fenómeno de la globalización va acompañado de una ideología políticoeconómica de corte neoliberal que considera positiva la globalización económica y del mercado; pero no ve tan necesaria la globalización política, ya que considera que el mejor funcionamiento de la economía es aquél en el que hay pocas interferencias políticas.

- Toma de conciencia de los problemas medioambientales: La humanidad toma conciencia de las amenazas que se ciernen sobre el medio ambiente a causa de la incontrolada actividad económica, pero aún no se han puesto medios suficientes para remediarlo. Las conferencias mundiales que se convocan para abordar el tema no cuentan aún con el apoyo decidido de los países más poderosos y desarrollados, que además son los que contaminan más.

- Consolidación del "estado del bienestar": La consolidación del "Estado del bienestar” entre la mayoría países desarrollados (educación y sanidad gratuita, pensiones de jubilación), son fundamentales en los avatares del desafío, aunque en algunos países aparecen ciertos indicios de recesión de los logros conseguidos.

Todas estas políticas de globalización ha traído consigo la articulación de conocimientos y especializaciones en las distinctas ciencias como en: la salud, cibernética,

1 Sancho Gil, Joana (1996). Educación en la era de la información”. Revista Cuadernos de Pedagogía no 253. Barcelona.

2 Homi Bhabha (1994) The location of culture, Routledge, Londres. 
teorías ambientales basado en la educación planetaria, teoría de la identidad -planetaria, revalorización de las culturas ancestrales, política interculturalidad y otros. La razón de ser de estas tendencias es la exploración y comprensión de conocimientos específicos aún vigentes en el mundo. Proceso de articulación. La demanda del reconocimiento del uno y el otro. Llamadas en el mundo académico. Teoría de la otredad, teoría de los derechos humanos y teorías del desarrollo sostenible. En la educación actual todas estas teorías son desafíos de capital humano como materia intelectual y consigo la mirada crítica de transformación social y colectiva.

Específicamente, el salto medular de la civilización desarrollada en los momentos actuales, que según la tesis de Castells, ha sido y sigue siendo un proceso de descubrimiento de conocimientos científicos para hacer cosas de una manera más sofisticada. Donde la principal característica se muestra en la aplicación de conocimiento e información a aparatos de generación conocimiento y procesamiento de información/ información. Asimsimo, también los usuarios han mostrado un aprendizaje constante en la creación y reconfiguración de redes de interacción como retroalimentación en la introducción de nuevas tecnologías, en la utilización y desarrollo en nuevos campos y esto se hizo más rápido en el nuevo paradigma tecnológico.

Tomas Kuhn, explica, en su obra las Estructuras de las Revoluciones Científicas, la esencia de la transformación de las revoluciones tecnológicas actuales y su interacción con la economía y con la sociedad, a esto muchos sociólogos los llaman actualmente como realidad societal. Y otra de las características que plantea sobre los rasgos que constituyen el núcleo del paradigma de la tecnología de la información es la configuración de la educación tecnológica: educación a distancia, cibernética, NET WEB. Dentro de este contexto de la globalización como horizonte de sentido y como aproximación hacia la sociedad del conocimiento, nos hace ver al paradigma tecnológico organizado en torno a las tecnologías de información, capacidad de penetración de los efectos de las nuevas tecnologías y la lógica de interconexión ${ }^{3}$ , ha codificado el proceso actual de transformación de la sociedad en su conjunto como redes interactúantes. Complejidad. Esto ha modificado las formas del comportamiento de la cultura, política, economía, sociedad, específicamente, de la educación. La educación bajo el enfoque de comptencias y bajo el enfoque de estimulación temprana en los niños por ejemplo.

Otra forma de comprender a los problemas de la globalización es desde las esperas de la tendencia de mundialización o sociedad del conocimiento, que actualmente se define como combinación de información, experiencia, contexto, interpretación y reflexión, pero esto desde una perspectiva sofisticada ha sido ya vista por Platón, desde los grados del conocimiento como: conocimiento por experiencia, por opinión, por espíteme y por intuición racional (conocimiento supremo) ${ }^{4}$.

Así como señala Savater, se mundializa los intereses económicos, pero no logra mundializarse los intereses de los derechos básicos de las personas. ${ }^{5}$ Una ideología desigual. Estas situaciones del entorno obedecen al contexto de la competitividad económica globalizado, específicamente empresarial, que ha sido modelo según los diagnósticos de Luis Alvarado. Este autor del artículo "Análisis actual del entorno económico" desarrolla la operatividad de la innovación educativa desde los principales objetivos como el capital intelec-

4 Carlota Pérez y otros: temas de reflexión dentro del curso de Gestión del Conocimiento en 2010. PUCP.

5 Lecturas personales sobre las ideas filosóficas de Platón: Teoría de las ideas

6 Véase a las citas, que recoge de Savater, Zygmud Bauman en su ensayo: Tiempos líquidos. 
tual de innovación que permiten identificar las competencias y las capacidades esenciales.

Esto permite detectar nuevas miradas de gestión del conocimiento, que desde nuestra perspectiva en el camino hacia la educación basada en la crítica es hacer una autocritica y generar la creatidad. Es decir, la educación planetaria es una propuesta humanizadora de personas para personas, esto significa la Era de ciencias humanas, o, educación humanista.

\section{Problemática social y el nuevo rol de las organizaciones educativas}

Los problemas sociales y las nuevas tareas en la organización educativa en la actualidad obedecen a los impactos y desafíos de las tendencias del desarrollo a gran escala a nivel mundial, esto nos exige a dar respuestas a las demandas de las instituciones educativas, pero la reorganización de la educación como tal no dependerá solo de la intencionalidad de cambio y de uso de las herramientas tecnológicas, sino tomará en cuenta a las nuevas teorías como la complejidad, que desde nuestra perspectiva es el símbolo de "camino a la era planetaria”.

Siguiendo nuestro periplo desde la perspectiva de la Era planetaria de la educación, ante la deshumanización de la propia sociedad, encontramos la denuncia puesta por Edgar Morín, que plantea un paradigma de concientización e identificación con el mundo. Identidad terrenal. Para ello, solicita el autor una educación planetaria explicada en la Teoría de la complejidad: pensamiento complejo (ciencias humanas) y sistemas complejos (ciencias fácticas).

La filosofía de Morín, refiriéndose a la educación concientiza y sensibiliza al ser humano, no solo cuando habla sobre lo que debe ser la educación en el futuro, sino lo que debemos hacer como seres humanos del planeta, es decir, pensar en ella para cuidar y cuidarnos entre humanos. Para eso elabora un proyecto de humanización y lo llama civilización planetaria y ciudadanía cosmopolita ${ }^{6}$.

Esta tesis discutida por muchos pensadores actualmente, nos hace ver la visión holística que entraña la relación del ser humano con la naturaleza (ambiente) y el planeta (tierra), esto no es sino un proyecto del hombre en comprender desde los sistemas complejos articulados entre sí. Que se debe tomarse en cuenta en las instituciones educativas ya que son núcleos de interacción académica, formación, social y generación de conocimientos. Por ello el rol de las instituciones cual sea su fase de desarrollo deben aplicar con mucha mesura las teorías nuevas con la finalidad de cambiar las formas de ver la realidad educativa y evitar equívocos constantes como se ha constatado históricamente.

El análisis del recorrido histórico de la civilización del hombre por el hombre para Morín es un paso hacia la sensibilización para con la naturaleza, y esto parte desde la educación planetaria, donde el concepto de identidad toma fuerza esencialmente. Es decir, es el ser mismo que se traduce en la dignidad configurada y defendida por los derechos humanos en la actualidad -la esencia de la vida-, paso a elaborar para la educación de calidad desde el enfoque del gestión del conocimiento.

Para nuestra mirada la cultura occidental siempre se ha desarrollado bajo el paradigma de ilusión como impulso del progreso y el desarrollo. Y el mismo concepto ha sido identificado como la marcha de la historia transcurrida a una historia cognositivista, es decir, inventada o transcrita por un gru-

6 Diógenes Laercio, en el siglo $\mathrm{V}$ aC., señalaba que como seres cosmopolitas habría que buscar un hombre honesto e identificarnos con la naturaleza cósmica, donde la antorcha incidida simbolizaba nuestra razón y esta era una herramienta para llegar a la verdad y justicia, y una educación sólida basada en la libertad. Interpretación personal. 
po de personas, que se han mantenido en el poder durante años -cual fuere la (s) ideología (s)- han elaborado una historia fallida sin criterios de verdad. Pues, por estas razones muchas veces la verdad se vuelve encrucijada, es decir sin salida. Como la teoría de las cavernas de Platón. Por ello, para evitar esto debemos plantear un proyecto educativo.

Otro de los puntos a considerar dentro del marco del rol institucional para la educación es el concepto desarrollo que debe asegurar el progreso. Señala Morín al enfatizar desde el marco semántico dos aspectos: por un lado considera que es un mito global en el que las sociedades se industrializan ... buscando la utopía felicidad, y por otro lado, la concepción reduccionista donde el crecimiento económico es el motor necesario para todo desarrollo (... ) concepción que ignora los problemas humanos de la identidad, de la comunidad, de la solidaridad y de la cultura.

Sean estas u otras situaciones en la sociedad de conocimiento, cuando se trata de la cultura educativa, se requiere una transformación radical ante el egocéntrismo dogmatico, economicismo empresarial polarizada de propagandas de consumo masivo y la formación de la mala conciencia a la juventud en la educación, especialmente si hablamos de la realidad peruana.

Por ello, "el hombre vive en el sombrío y busca el cambio en la situación nostálgica de la caminata en la tierra patria"7. Esta poética afirmación permite a discernir y comprender el paradigma de gestion de conocimiento para comprender mejor lo que ocurre en la cotidianidad humana con miras hacia la calidad educativa.

Para tal efecto, elaborar el proyecto educativo a largo plazo se debe pensar y diseñar una política bajo el paradigma de capital in- telectual, humano y organizacional, partiendo de juicios críticos y recogiendo la teoría de los valores y principios éticos que conduzcan hacia la planetarización de la identidad y comprensión de las cosas para hacer y ser mejores ciudadanos del mundo. Donde la capacidad intelectual sea valorada por lo que es en sí mismo, es decir por su esencia trascendental e intangible y no tanto desde su tangibilidad cosificada. Algunas ideas propositivas para generar cambio ${ }^{8}$ :

- La educación desde la teoría de complejidad permitirá a los agentes a tomar decisiones y actuar apropiadamente, porque parte de la teoría holística articulada.

- La reformulación del concepto Gestión y Conocimiento desde el enfoque filosófico crítico permitirá a que la tendencias de Acción práctica se situé en la conciencia del capital humano-intelectual.

- El paradigma aleatorio en la gestión permitiría la reorganización de modelos tradicionales que sería la fortaleza del modelo humanista.

- El replanteamiento del concepto de persona en la educación llevaría a elaborar un proyecto a largo plazo con miras hacia la sensibilización y valoración de la existencia en sí fortalecida por la esencia que es el ser en cuanto ser.

- La comprensión y el entendimiento de principios éticos y valores morales conduciría a los educadores y educandos a tener noción y práctica lógica de los mismos en la era planetaria.

- Analizar y desarrollar del proyecto nacional de educación y la ley de la educación universitaria desde el enfoque de Gestión del conocimiento generaría una política de calidad y competencia para el desarrollo hu-

7 Romero, Ruth. Ensayos filosóficos para religar los saberes actuales, p. 121. Proyecto en proceso para su publicación.

8 Op.cit., p. 178. Ideas propias para elaborar un diagnóstico de una teoría educativa en la actualidad. 
mano y responsabilidad social, puesta a la acción práctica.

En consecuencia, de manera específica podemos también apreciar de manera detallada algunos problemas reales dentro de las organizaciones educativas y políticas de la educación desde la perspectiva políticas públicas del estado, partiendo de esta frase: Nadie da lo que no tiene. Esta tesis es clara en la conciencia de muchas personas cuando afirman que no dan lo que saben o tienen, sin embargo, no saben ni tienen. Por tanto, se genera, pues, una encrucijada sin salida cuando se habla de manera específica sobre los planes o diseños curriculares en la educación universitarai o de básica regular.

Otro de los aspectos fundamentales de la educación en la actualidad y dentro del rol de las instituciones educativas es la siguiente cuestión: ¿Por qué el actual Diseño Curricular Nacional es tan ajeno a los docentes de nuestro país? Seguirá la conducta del "cumplimento", "cumplo y miento", es decir, cumplir con los formalismos del Diseño Curricular, pero en el aula, no realizar lo que realmente se plantea en el currículo, sino trabajar con lo que realmente se conoce y de la manera en la que fueron formados, es decir, actuar de manera coherente con la teoría implícita.

La opinión pública e incluso el Ministerio de Educación, debido al impacto que tienen en la sociedad los resultados de diferentes evaluaciones, específicamente en la educación básica regular por ejemplo (PISA, LLECE, EN 2004, etc.) sobre la calidad de los aprendizajes han resposabilizado fundamentalmente a los docentes de los bajos rendimientos, por su lado la organización gremial responsabilizó al gobierno del turno y al sistema generando un desencuentro, en nuestra opinión, innecesario que no conlleva una búsqueda de una solución racional y crítica. Por lo que "La paradoja praxiológica que se refleja duramente en los muy precarios aprendizajes de los esco- lares peruanos consiste en que sus profesores son formados durante cinco años para que intenten «enseñar» lo que, estrictamente, no saben" (Piscoya 2005: 95).

Por otro lado, en la última década, en nuestro país, mucho se ha escrito y discutido sobre la calidad de la educación universitaria o no universitaria y, desde diferentes perspectivas, todos coinciden que aún estamos lejos de alcanzar los estándares de la ansiada calidad. Por ejemplo, el Banco Mundial (2006) sostiene que se hemos logrado un gran progreso en la cobertura del sistema educativo. Pero los aprendizajes de los niños y el aprestamiento de los jóvenes para la vida dejan mucho que desear y que estos no solo son de bajo nivel, sino que están muy mal distribuidos en la sociedad.

Algunos responsabilizan de la baja calidad de enseñanza al escaso presupuesto para el sector educativo y el escazo de organización estructural de las instituciones, otros no creen en las estructuras ni en las organizaciones ni en los sistemas, pues consideran que el profesor en el aula está solo y sostienen que "él es el responsable, más allá de los dualismos y de los sistemas externos de normas, de su intervención; él debe interpretar la situación con sus estudiantes sin interferencias, ni condicionamiento aparentes para que, de ese encuentro, quizá germinen semillas que nunca se sembraron porque la enseñanza es una interacción subjetiva e incierta" (Flores 1999: 186)

El cumplimiento de una programación curricular no refleja, necesariamente, el proceso que ocurre en el fondo del ser de cada docente, tampoco lo que ocurre en el ser de cada educando, sino es más complejo. Una filosofía del currículo compleja es la mediación entre la teoría y la realidad de la enseñanza, un plan de acción que desarrolla el profesor con sus alumnos en el aula, una pauta ordenadora del proceso de enseñanza. Y cada perspectiva, 
cada teoría genera una propuesta de currículo diferente desde la posición plural compleja.

La pregunta central es icuál es la Teoría que ha construido el docente a lo largo de su vida para se reconocido como tal o para ser un buen docente en las prácticas de la enseñan$\mathrm{za}$ ? no se trata solamente de lo que ha estudiado como teorías curriculares en su formación académica, sino la manera en la que esos conocimientos fueron procesados y cómo reformularon su concepción de educación y cómo aplica en los escenarios del encuentro con el otro que es el estudiante. Esta concepción será la teoría implícita y se concretará en un modelo explicito y a la vez guiará la práctica curricular, muchas veces lejana del modelo curricular oficial que las insitcuiones poseen cada entidad educativa de formación o de enseñanza.

Conocer los aspectos formativos de la vida de los que hoy ejercen la docencia universitaria en nuestro país es una variable de investigación necesaria para entender y explicar, por ejemplo: por qué un modelo curricular basado en competencias, con algunas características de interpretativo cultural y rasgos de crítica no tiene el éxito esperado tanto en los aprendizajes, como en el desarrollo de los estudiantes; menos aun en la realización profesional de los docentes.

La experiencia educativa implícita, formada durante años, la que hoy poseen los que ejercen la docencia en nuestro país, ya sea en la Universidad, en las Escuelas Normales o en los Institutos Superiores Pedagógicos no hizo más que sistematizar el modelo tecnológico de los ya traían, esa "semilla", de la escuela. Mientras que para otros, que vivenciaron modelos curriculares más clásicos, la formación se constituyó en el aprendizaje de un modelo que supuestamente tendría que haber modificado el esquema clásico que traían.

En los años 80 se realizaron cambios curriculares, en los cuales se introduce un mo- delo interpretativo cultural bajo la forma de un enfoque constructivista. Proceso que fue identificado como una propuesta política, más que pedagógica y fue implementada sin haber realizado una preparación previa en el magisterio y en las universidades.

En este contexto los resultados no son sorprendentes: "el Perú ha participado en las pruebas internacionales PISA 2000 y LLECE. La prueba PISA 2000 es tal vez más útil como patrón, pues incluye una gama más amplia de países. El desempeño peruano en ella (tomando juntos, como promedio simple, la capacidad global de lectura, la de matemática y la de ciencias) fue peor que el de cualquier otro país participante, incluyendo a los demás países en vías de desarrollo de la muestra. (Banco Mundial 2006: 3), esto es una muestra clara de una calidad carente de conocimientos que se presencian en la experiencia universitaria.

Muchos responsabilizaron de esta realidad a los docentes, y esa conclusión es muy injusta porque como lo sostiene el Banco Mundial: "El Perú era uno de los países más pobres que participaban en PISA 2000, y también uno de los que más recientemente habían transitado hacia la alfabetización masiva. De todos los países incluidos en PISA 2000, el Perú tenía la segunda tasa más alta de jóvenes (la población de 0-14 sobre la de 1564). Si tomamos en cuenta simultáneamente estos tres factores de desventaja, el desempeño peruano en PISA 2000 solo fue algo peor de lo esperado"

Por otro lado, la evaluación aplicada a los docentes realizada en el año 2002 muestra un perfil general de la formación que es calificado por Luis Piscoya del siguiente modo: "los muy bajos rendimientos académicos de 95,219 docentes peruanos en las pruebas de suficiencia profesional-2002, segmentadas en 14 áreas de especialización, permite deducir que no sólo los programas de capacitación do- 
cente están severamente desorientados sino que, principalmente, el sistema de formación docente constituido por los institutos superiores pedagógicos, dirigidos académicamente por Dinfocad, y las facultades de Educación de las universidades atraviesa por una crisis cuyos inicios tienen no menos de 25 años de antigüedad. Los síntomas más ostensibles de esta crisis son el «pedagogicismo» que afecta a los centros de formación magisterial y, su complemento, el gremialismo corporativista que se expresa en la trayectoria del sindicato nacional de docentes". (Piscoya 2005: 95).

$\mathrm{El}$ docente es el componente principal de la ejecución de la concepción de enseñanza y aprendizaje del modelo curricular en aplicación. De su actuación depende el éxito de los resultados educativos y de los resultados institucionales. Es el responsable de seleccionador las técnicas y medios disponibles para alcanzar los objetivos previstos. Es quien hace práctica la teoría y el modelo curricular haciendo un epogè en la formación del nuevo educando.

En definitiva, una de las condiciones necesarias, quizá la más trascendental, para que un modelo educativa sea eficaz, eficiente, pertinente y trascendente es que los docentes que la aplicarán deban conocer primero el contenido y la organización lógica de lo que se pretende aplicar, en caso contrario saldrá, de manera natural, el modelo implícito academicista que fue formado a lo largo de su vida. ¿Se les puede responsabilizar por ello?

Un modelo educativo (currículo) puede haber sido concebido por los más expertos especialistas, conocedores de la realidad, puede tener todas las características técnicas en su construcción, pero si los docentes que lo implementan no le encuentran sentido, entonces, será solamente un documento más, un esfuerzo que no toma en cuenta la trascen- dencia del docente en la selección del modelo curricular y el proceso del diseño curricular en la institución académica.

No obstante, la sociedad educativa bien gestionada debe tener una mirada a la tesis realista y crítica de Bauman, para evitar que se siga creándose en la historia los proyectos educativos y otros, sin futuro y mal planificado y organizado. Señala Bauman, referiendose a la sociedad actual que "el paso de la fase de la sólida de la modernidad a la liquida. Es decir a una condición en las que las formas de organización ya no pueden mantener su forma por más tiempo, porque se descomponen y se derriten antes de que se cuente con el tiempo necesario para asumirlas, y una vez asumidas, ocupar el lugar que se le ha asignado".

Esto significa que, lo que acontece en nuestra cotidianidad educativa es una ilusión, delirio, angustia vencidos por el corto plazo, donde los proyectos y la propia gestión han tenido una carencia de elementos lógicos, sólidos y válidos. Llevando asi al error en la organización y gestión, donde la sensibilidad y la responsabilidad, y otros deja de ser importante y prioritarios.

El conocimiento es un paradigma gnoseológico dentro de la filosofía, donde separa del conocer, saber, hacer, y ser; esto significa estar en contacto directo con las cosas externas o internas en el mundo físico y no físico. Que en el ámbito empresarial administrativa y organizativa es la operatividad de la tangible o intangible. Por ello, el conocimiento es inherente a la persona, porque la persona por el hecho de poseer su dignidad presenta derechos propios como tales, por tanto es un ser humano. El ser humano por naturaleza propia desea conocer para satisfacer sus necesidades. En este querer satisfacer, nace de la curiosidad $y$ esto es innato, porque presenta una racionalidad que le permite generar y crear formas de

9 Bauman, Z., (2007) Tiempos líquidos: Vivir en una época de incertidumbre, México, Ed.TusQuests. Vol. 72. 
organización o vivir de manera organizativa en una sociedad de cultura; dentro del marco de cultura está el aspecto fundamental que es la educación, por ello, para las personas siempre fueron básicos y primordiales, tanto en espacios micro, medianos, o macros en la existencia social humana.

\section{CONCLUSIONES}

- Se concluye que en la sociedad de conocimiento la globalización es producto de la modernidad y sus desafíos que no solo han generado un cambio sino una trasformación significativa esencialmente.

- El paradigma aleatorio en gestión racional ha permitido la reorganización de modelos tradicionales para fortalecer a los nuevos modelos humanistas.

- El replanteamiento del concepto de persona en la educación ha llevado ha elaborar un proyecto de sensibilización y valoración del concepto del educar dentro de las tendencias de ser humano por el ser humano.

- La teoría de la complejidad con pespectivas de la interculturalidad ayuda a desarrollar el paradigma de capital intelectual con esencias de trascendentalidad e intangibilidad.

\section{RECOMENDACIONES}

- Se debe crear un plan curricular educativo universitario de acuerdo a la realidad actual considerando la posición humanista compleja.

- Los paradigmas de mundialización y planetaricación debe ser tomada como un nuevo enfoque para gestionar el conocimiento en la sociedad global y local.

- La política universitaria educativa debe diseñar una enseñanza y producción de conocimiento sobre la base de la generacion de la investigación.

\section{REFERENCIAS BIBLIOGRÁFICAS}

1. ALVARADO, Acuña. (2005) Análisis actual al entorno económico. Chile. Universidad Católica del norte.

2. CADE -38, (2001) "Perú: ¿en qué país queremos vivir? La apuesta por la educación y la cultura. Tomo II Cultura. 38 CADE. Lima.

3. CANO, Elena. (2003) Organización, calidad y diversidad. Colección Aula Abierta. Madrid. Editorial La Muralla. CANAL S.A.

4. CASTELLS, Manuel. (2000) La Era de la Información. Economía, sociedad y cultura. Vol.1. 5ta. Edición. Massachusetts. Alianza Editorial.

5. DELORS, J. (1997) La educación encierra un tesoro. Informe de la Comisión,. Internacional sobre la educación sobre el siglo XXI. Santillana Ediciones-UNESCO.

6. HALLAK, Jaques. Educando para el futuro.

7. MORÍN Edgar, CIURANA, Emilio $y$ MOTTA, Raúl. (2003). Educar en la era planetaria. Nueva York. Edit. Gedisa.

8. 8. BANCO MUNDIAL. (2006). Por una educación de calidad para el Perú Estándares, rendición de cuentas y fortalecimiento de capacidades. Asociación gráfica educativa. Lima

9. 9. CHIROQUE, Sigfredo (2007) Situación del docente en el Perú. Instituto de Pedagogia Popular IPP Lima

10. 10. FLOREZ, Rafael, (1999) Evaluación pedagógica y cognición. McGraw-Hill. Bogotá

11. 11. PISCOYA, Luis (2005) Cuánto saben nuestros maestros. Una entrada a los diez problemas cardinales de la educación peruana. UNMSM, Fondo Editorial; COFIDE: 2005. Lima

12. 12. VVAA. (2009) Gestión del conocimiento. Una exigencia de la educación actual. INFODEM Lima, Industria Gráfica San Remo S.A.C. 